\title{
Testing for heritable thrombophilia in acute venous thromboembolism
}

\author{
Eric Tseng MD, Rita Selby MD
}

Cite as: CMAJ 2017 July 4;189:E891. doi: 10.1503/cmaj.160062

1

Heritable thrombophilias are inherited disorders that increase the risk of venous thromboembolism (VTE)

Common heritable thrombophilias include factor V Leiden ( 1 in 20 white Europeans are heterozygous) and prothrombin G20210A (1 in 50 white Europeans are heterozygous), and uncommon ones include protein $\mathrm{C}$ deficiency, protein $S$ deficiency and antithrombin deficiency (Appendix 1, available at www.cmaj. ca/lookup/suppl/doi:10.1503/cmaj.160062/-/DC1). ${ }^{1}$ These disorders are commonly included in panels of testing for heritable thrombophilia.

2

Tests for heritable thrombophilia should not be done during an episode of acute VTE, as they are often inaccurate in this context Protein $\mathrm{C}$, protein $\mathrm{S}$ and antithrombin levels are often low during acute VTE and in the presence of anticoagulants, and do not necessarily reflect true deficiency. ${ }^{2}$ The presence of heritable thrombophilia also does not affect initial anticoagulant choice or dose. ${ }^{3}$

The presence of heritable thrombophilia should not affect the duration of anticoagulation in acute VTE

The presence of heritable thrombophilia does not add independent predictive value to estimates of recurrence of VTE and therefore should not affect treatment duration in acute VTE. ${ }^{1,3}$ The risk of recurrent VTE - and the decision to extend anticoagulant therapy - is more strongly dependent on clinical risk factors, such as lack of provoking factors, male gender, persistently elevated D-dimer, or current active cancer, each of which confer a higher risk of recurrence. ${ }^{4}$

Routine testing for heritable thrombophilia in relatives of patients with VTE has not been shown to affect clinical outcomes ${ }^{5}$

In some circumstances (e.g., starting oral contraception), testing for a particular heritable thrombophilia may be considered in an asymptomatic patient with a family history of VTE and a known heritable thrombophilia. However, a hematologist or thrombosis expert should discuss the benefits and limitations of such testing with the patient before the tests are ordered. ${ }^{3}$

Testing for heritable thrombophilia is expensive and provides low value to the health care system

A recent cost analysis of testing in Canada for factor $\mathrm{V}$ Leiden and prothrombin gene mutation after a first unprovoked VTE concluded that reduction in testing would result in net economic savings. ${ }^{6}$ These savings would be driven by the high cost of testing, and by changes in physician-ordering practices of anticoagulants, based on test results that have not been shown to improve clinical outcomes. ${ }^{6}$

\section{References}

1. Christiansen SC, Cannegieter SC, Koster T, et al. Thrombophilia, clinical factors, and recurrent venous thrombotic events. JAMA 2005;293:2352-61.

2. Marlar RA, Gausman JN. Laboratory testing issues for protein C, protein S, and antithrombin. Int J Lab Hematol 2014;36:289-95.

3. Baglin T, Gray E, Greaves M, et al. British Committee for Standards in Haematology. Clinical guidelines for testing for heritable thrombophilia. Br J Haematol 2010; 149:209-20.

4. Agnelli G, Becattini C. Risk assessment for recurrence and optimal agents for extended treatment of venous thromboembolism. Hematology Am Soc Hematol Educ Program 2013;2013:471-7.

5. De Stefano V, Rossi E. Testing for inherited thrombophilia and consequences for antithrombotic prophylaxis in patients with venous thromboembolism and their relatives. A review of the Guidelines from Scientific Societies and Working Groups. Thromb Haemost 2013; 110:697-705.

6. CADTH optimal use report: Effectiveness of factor $V$ Leiden and prothrombin mutation testing in patients presenting with a first unprovoked venous thromboembolic episode: a systematic review and economic analysis. Vol. 4, No. 1A. Ottawa: Canadian Agency for Drugs and Technologies in Health; 2015. Available: www.cadth .ca/media/pdf/OP0517_Thrombophilia_Science_Report .pdf (accessed 2016 June 14).

Competing interests: Rita Selby reports grants from Boehringer Ingelheim, outside the submitted work.

This article has been peer reviewed.

Affiliations: Department of Medicine, Division of Hematology (Tseng, Selby); Department of Laboratory Medicine and Pathobiology (Selby); University Health Network and Sunnybrook Health Sciences Centre, University of Toronto, Toronto, Ont.

Correspondence to: Rita Selby, rita.selby@sunnybrook.ca 